\title{
Docking Studies of Binding of Ethambutol to the C-Terminal Domain of the Arabinosyltransferase from Mycobacterium tuberculosis
}

\author{
Guillermo Salgado-Moran, ${ }^{1}$ Rodrigo Ramirez-Tagle, ${ }^{2}$ Daniel Glossman-Mitnik, ${ }^{3}$ \\ Samuel Ruiz-Nieto, ${ }^{4}$ Pran Kishore-Deb, ${ }^{5}$ Marta Bunster, ${ }^{6}$ and Francisco Lobos-Gonzalez ${ }^{6}$ \\ ${ }^{1}$ Departamento de Ciencias Químicas, Facultad de Ciencias Exactas, Universidad Andrés Bello, Sede Concepción, \\ Concepción 4070000, Chile \\ ${ }^{2}$ Universidad Bernardo O'Higgins, Laboratorio de Bionanotecnología, General Gana 1780, Santiago 7490000, Chile \\ ${ }^{3}$ Laboratorio Virtual NANOCOSMOS, Centro de Investigación en Materiales Avanzados, Miguel de Cervantes 120, \\ Complejo Industrial Chihuahua, 31109 Chihuahua CHIH, Mexico \\ ${ }^{4}$ Facultad de Medicina, Universidad Diego Portales, Avenida Ejército 233, Santiago 7490000, Chile \\ ${ }^{5}$ Pharmaceutical Chemistry Division, Institute of Pharmaceutical Sciences and Centre of Advanced Study in Pharmaceutical Sciences \\ (UGC-CAS), Panjab University, Chandigarh 14, India \\ ${ }^{6}$ Laboratorio de Biofísica Molecular, Departamento de Bioquímica y Biología Molecular, Facultad de Ciencias Biológicas, \\ Universidad de Concepción, Casilla 160-C, Concepción 407000, Chile
}

Correspondence should be addressed to Daniel Glossman-Mitnik; daniel.glossman@cimav.edu.mx

Received 24 October 2012; Revised 30 January 2013; Accepted 20 February 2013

Academic Editor: Joaquin Campos

Copyright (C) 2013 Guillermo Salgado-Moran et al. This is an open access article distributed under the Creative Commons Attribution License, which permits unrestricted use, distribution, and reproduction in any medium, provided the original work is properly cited.

\begin{abstract}
The binding of ethambutol to the C-terminal domain of the arabinosyltransferase from Mycobacterium tuberculosis was studied. The analysis was performed using an in silico approach in order to find out, by docking calculations and energy descriptors, the conformer of Ethambutol that forms the most stable complex with the C-terminal domain of arabinosyltransferase. The complex shows that location of the Ethambutol coincides with the cocrystallization ligand position and that amino acid residues ASH1051, ASN740, ASP1052, and ARG1055 should be critical in the binding of Ethambutol to C-terminal domain EmbC.
\end{abstract}

\section{Introduction}

Ethambutol (EMB) is an antimycobacterial drug used extensively for the treatment of tuberculosis caused by Mycobacterium tuberculosis (TB). EMB is bacteriostatic and it is used worldwide for tuberculosis therapy in combination with isoniazid, pyrazinamide, and rifampicin.

The EMB target is the biosynthesis of the cell wall, by inhibiting the synthesis of both arabinogalactan and lipoarabinomannan (LAM), which are essential components of the unique cell envelope of the pathogen TB. Biosynthesis of LAM involves a series of membrane embedded arabinosyltransferases: EmbA, EmbB, and EmbC. Recent works show that mutations in EmbC that reduce its arabinosyltransferases activity result in increased sensitivity to $\mathrm{EMB}$, and there exists a direct correlation between EmbC activity and EMB resistance, as well as between EmbC activity and the size of the LAM species produced, confirming that EmbC is one of the cellular targets of the EMB action [1]. It has been reported that (Escuyer et al., 2001), in M. smegmatis, EmbA, B, and C are necessary for the mycobacterium to build its cell wall. The resistance to EMB has been reported in as many as $4 \%$ of the clinical isolates of TB and it is prevalent among multidrugresistant strains [2]. Some mutant proteins from the Emb family $(\mathrm{ABC})$ have been isolated in ethambutol-resistant Mycobacterium tuberculosis (Srinivas V Ramaswamy, Amol G. Amin, Servet Goksel, Charles E. Stager, Shu-Jun Dou, Hana el Sahli, Soraya L. Moghazeh, Barry, N. Kreiswirth, 
James M. Musser, "Molecular Genetic Analysis of Nucleotide Polymorphisms Associated with Ethambutol Resistance of Human Isolates of Mycobacterium tuberculosis," Antimicrobial Agents and Chemotherapy, 44(2) (2000) 326-336). The sequence similarity among the proteins of this family (UNIPROT Data Base) is close to 57\%. Until today only an extracellular segment of the protein has been expressed, purified, and its 3D structure determined. It corresponds to the C-terminal [2] region of the polypeptide chain of $390 \mathrm{aa}$, and some of the mutations associated with resistance to ethambutol are located in this zone.

Despite the availability of modern antibiotics, infectious diseases are responsible for nearly one-third of human deaths worldwide, and bacterial resistance is still an urgent problem. Bacterial resistance to antibiotics is the inevitable consequence of the overuse of Antimicrobial agents. Computational strategies for structure-based drug discovery offer a valuable alternative to the costly and time-consuming process of random screening.

Docking calculations have been applied in pharmaceutical research for nearly two decades. Virtual screening on protein templates, which differs from molecular similarityand ligand-based virtual screening methods, provides an opportunity for de novo identification of active compounds, without bias towards known hits or leads [3].

In order to understand the action of ethambutol as a pharmaceutical, we have used the structure of the C-terminal domain of EmbC which was crystallized in presence of a sugar ligand associated with a potential substrate recognition binding site. Using this information we have built a docking model between EmbC domain and and the ligand ethambutol. The top binding poses of EMB in the C-terminal domain of the TB were selected considering the binding energies. Considering that some of the point mutations have been observed in this segment of the protein, we will analyze the relationship with their possible resistance to ethambutol.

\section{Experimental}

2.1. Molecular Docking Study. Molecular docking in silico experiments were performed with GLIDE v5 XP docking program (Schrodinger Inc.) $[4,5]$ using a Dell Precision workstation T3400 running in an Intel Core2 Duo Processor, 4 GB RAM, 250 GB hard disk, and Nvidia Quadro FX 4500 graphics card.

2.2. Preparation of Protein. The three-dimensional coordinates of the C-terminal domain of arabinosyltransferase (PDB code: 3pty (http://www.rcsb.org/) were downloaded, refined, and prepared using Schrodinger protein preparation wizard tool (GLIDE), which performs the following steps: assigning of bond orders, addition of hydrogens, optimization of hydrogen bonds by flipping amino side chains, correction of charges, and minimization of the protein complex. All the bound water molecules, ligands, and cofactors were removed (preprocess) from the protein which were stored (as. Mae) format. The program also neutralized the side chains that are not close to the binding cavity and do not participate in salt bridges. This step is then followed by restrained minimization of the cocrystallized complex, which reoriented the hydroxyl groups of the side chains diminishing potential steric clashes. The complex obtained was minimized using OPLS_2005 force field [6] with Polak-Ribiere Conjugate Gradient (PRCG) algorithm. The minimization was terminated either by completion of 5,000 steps or after the energy gradient converged below $0.05 \mathrm{kcal} / \mathrm{mol}$.

2.3. Preparation of Ligands. The cocrystallized ligand AFO1 (octyl alpha-D-arabinofuranoside) was used as reference ligand throughout this study. The structure of ethambutol was built in the panel of Maestro and store (in. mae) format. LigPrep is a utility of the Schrodinger software suit that combines tools for generating 3D structures from 1D (Smiles) and $2 \mathrm{D}(\mathrm{SDF})$ representation, searching for tautomers and steric isomers, and performing geometry minimization of the ligands. Molecular Mechanics Force Fields OPLS_2005 was used with default settings.

2.4. Calculation of Prime MM-GBSA Descriptors. The Prime MM-GBSA approach [7] is used to predict the free energy of binding for a receptor and a set of ligands. MM-GBSA is an acronym for a method that combines OPLS molecular mechanics energies $\left(E_{\mathrm{MM}}\right)$, an SGB solvation model for polar solvation $\left(G_{\mathrm{SGB}}\right)$, and a nonpolar solvation term $\left(G_{\mathrm{NP}}\right)$ composed of the nonpolar solvent accessible surface area and van der Waals interactions. The total free energy of binding is then expressed as

$$
\Delta G_{\text {bind }}=G_{\text {complex }}-\left(G_{\text {protein }}+G_{\text {ligand }}\right)
$$

where

$$
G=E_{\mathrm{MM}}+G_{\mathrm{SGB}}+G_{\mathrm{NP}}
$$

The ligand in the unbound state is minimized in SGB solvent but is not otherwise sampled. In the calculation of the complex, the ligand is minimized in the context of the receptor whose coordinates are not allowed to move. The following descriptors were generated by the Prime MMGBSA approach:

MM-GBSA_DG_bind Ligand binding energy, $\Delta G_{\text {bind }}$ MM-

GBSA_E_complex Energy of the complex, $G_{\text {complex }}$

MM-GBSA_E_protein Energy of the receptor without the ligand, $G_{\text {protein }}$

MM-GBSA_E_Ligand Energy of the unbound ligand, $G_{\text {ligand }}$

To set up the calculation, the pose viewer file (generated after docking with GLIDE) was used to consider the receptor and the conformer. After choosing the receptor and the ligand, by using pose viewer file ( $p v$.maegz), the program Prime MM-GBSA that generates the descriptors was run with default options that were chosen to produce reasonable descriptors. The MM-GB/SA scoring along with the experimental binding affinities data of $\mathrm{hA}_{3} \mathrm{AR}$ is presented in Table 1 . 


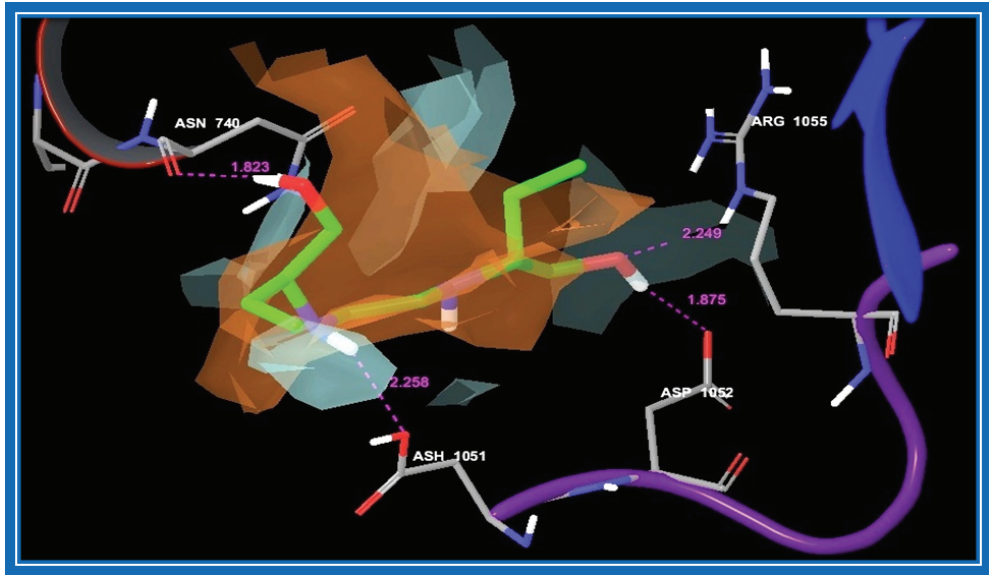

FiguRE 1: Binding orientation of most stable conformer $\left(\Delta G_{\text {bind }}=-53.208 \mathrm{kcal} / \mathrm{mol}\right)$ of Ethambutol. The hydrophilic and hydrophobic regions around the binding site are shown in cyan and orange, respectively.

TABLE 1: Free energy of binding of various conformers of Ethambutol with the receptor (arabinosyltransferase) (PDB ID: 3PTY)].

\begin{tabular}{|c|c|c|c|c|c|c|}
\hline $\begin{array}{l}\text { Conformers of } \\
\text { Ethambutol }\end{array}$ & $\begin{array}{c}\text { Prime_MMGBSA_ } \\
\text { Complex_Energy }\end{array}$ & $\begin{array}{c}\text { Prime_MMGBSA_ } \\
\text { Ligand_Energy }\end{array}$ & $\begin{array}{c}\text { Prime_MMGBSA_ } \\
\text { Receptor_Energy }\end{array}$ & $\begin{array}{c}\text { Prime_MMGBSA } \\
\text { DG_bind } \\
\left(\Delta G_{\text {bind }}=\mathrm{kcal} / \mathrm{mol}\right)\end{array}$ & $\begin{array}{l}\text { Prime_MMGBSA } \\
\text { DG_bind_Coulomb }\end{array}$ & $\begin{array}{c}\text { Prime_MMGBSA_- } \\
\text { DG_bind_vdW }\end{array}$ \\
\hline${ }^{\mathrm{a}}$ ligand_2 & 3238.91 & -4.697146797 & 3296.815925 & -53.2086913 & -13.86595 & -24.491161 \\
\hline ligand_12 & 3247.308 & -0.452434899 & 3296.815925 & -49.05529941 & -24.697604 & -20.847304 \\
\hline ligand_6 & 3250.839 & -4.53550489 & 3296.815925 & -41.44154781 & -19.49685 & -18.635326 \\
\hline ligand_10 & 3248.786 & -3.853684533 & 3296.815925 & -44.17586451 & 4.556659 & -22.396456 \\
\hline ligand_1 & 3245.459 & -6.414397018 & 3296.815925 & -44.94251808 & -12.807315 & -23.006476 \\
\hline ligand_8 & 3256.337 & 0.689291234 & 3296.815925 & -41.16800522 & -27.037333 & -13.034676 \\
\hline ligand_5 & 3251.727 & -0.257578354 & 3296.815925 & -44.83122776 & 9.086197 & -22.601608 \\
\hline ligand_3 & 3248.563 & -2.083358001 & 3296.815925 & -46.1690834 & -13.054461 & -19.804068 \\
\hline ligand_7 & 3252.905 & -0.676562682 & 3296.815925 & -43.23393085 & -19.953135 & -14.485878 \\
\hline ligand_14 & 3267.603 & 1.287304294 & 3296.815925 & -30.49988376 & -25.252316 & -12.033459 \\
\hline ligand_15 & 3262.401 & -2.120734728 & 3296.815925 & -32.29445194 & -31.278318 & -11.048768 \\
\hline ligand_4 & 3247.298 & -1.785731498 & 3296.815925 & -47.73175335 & -14.401013 & -22.61031 \\
\hline ligand_13 & 3257.699 & -7.07361709 & 3296.815925 & -32.0429963 & -21.157356 & -11.547959 \\
\hline ligand_11 & 3250.628 & -3.151238323 & 3296.815925 & -43.03688574 & 5.37957 & -22.90309 \\
\hline ligand_9 & 3248.916 & -4.634402555 & 3296.815925 & -43.26568145 & 7.795841 & -22.957128 \\
\hline ligand_16 & 3262.095 & -4.442765243 & 3296.815925 & -30.27776049 & -17.42324 & -14.158943 \\
\hline
\end{tabular}

${ }^{\mathrm{a}}$ Most stable conformer.

\section{Results and Discussion}

To recognize the hypothetical binding mode and interaction of ethambutol with crucial amino acid residues on the Cterminal domain of EmbC, molecular docking study was carried out by using the crystal structure of arabinosyltransferase (PDB ID: 3PTY). To investigate the ability of molecular docking to reproduce an experimentally observed ligand binding mode, the cocrystallized ligand AFO1 (octyl alpha-D-arabinofuranoside) was used as reference ligand and docked back into its binding site in the crystal structure of the arabinosyltransferase [8] using GLIDE XP docking program (Schrodinger Inc) $[4,5]$. The docking pose closely resembled the cocrystallized conformation with root-meansquare deviation (RMSD) of the polypeptide chain of $0.83 \AA$. The same docking protocol was used for the docking of ethambutol and the optimum conformation was used to analyze its interactions with amino acids residues in the binding site of the C-terminal domain in EmbC. Being more computationally demanding, the MM-GB/SA scoring gives far superior correlation with experimental activity data than standard docking scoring functions. The MMGB/SA methodology has also been much more reliable than docking for rank ordering of the poses. Hence, free energy of binding of various conformers of ethambutol with the receptor was calculated employing Prime MM-GBSA approach (Table 1). 

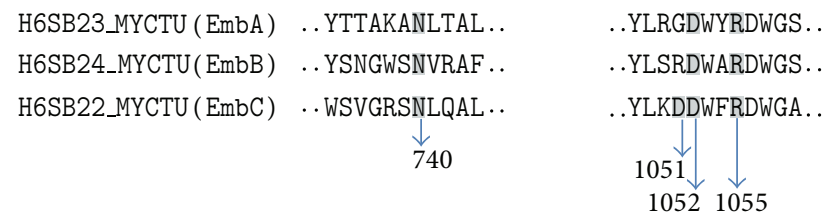

FIGURE 2: Preliminary sequence comparison of the C-terminal domain in EmbC with the same region in EmbB and EmbA using ClustalW.

TABLE 2: Electrostatic interaction energy $(\mathrm{kcal} / \mathrm{mol})$ and vdW interactions between the Ethambutol and crucial amino acid involved in ligand recognition.

\begin{tabular}{lcc}
\hline Amino acid residue & Electrostatic & vdW \\
\hline ASN740 & -1.873 & -3.213 \\
ASH1051 & 0.019 & -2.594 \\
ASP1052 & -6.433 & -0.732 \\
ARG1055 & -3.443 & -2.265 \\
\hline
\end{tabular}

The most stable conformer $\left(\Delta G_{\text {bind }}=-53.208 \mathrm{kcal} / \mathrm{mol}\right)$ of ethambutol (Figure 1) showed that its hydroxyl groups $(\mathrm{OH})$ were exposed to the hydrophilic region of the receptor (as indicated by cyan color on the hydrophilic map in Figure 1) and formed strong hydrogen bond interactions (short Acceptor-Donor distances) with crucial amino acid residues such as ASN740, ASP1052, and ARG1055 at distances of $1.823 \AA, 1.875 \AA$, and $2.249 \AA$, respectively. Similarly, one of the amino groups $(\mathrm{NH})$ exposed to hydrophilic region of the receptor formed an important $\mathrm{H}$-bond interaction with ASH1051 at a distance of $2.258 \AA$. The alkyl chain of the ethambutol with two ethyl substituents was found to be embedded in the hydrophobic region of the receptor as indicated by orange color on the hydrophilic map, which means that probably the binding of ethambutol is in strong competition with the natural substrate in the site. To analyze the possible ligand-receptor recognition mechanism in a more quantitative way, the individual electrostatic and vdW contributions to the interaction energy of each crucial residue of the receptor were calculated (Table 2). In particular, amino acid residues such as ASH1051, ASN740, ASP1052, and ARG1055 strongly stabilize the ligand-receptor complex (as indicated by the negative electrostatic interaction energy) because of the formation of strong hydrogen bonding interactions as shown in Figure 1. There is a strong possibility that ethambutol occupies a key position in the binding site of the C-terminal of arabinosyltransferase (EmbC) which would avoid the binding of a substrate.

Preliminary sequence comparison of the C-terminal domain in EmbC with the same region in EmbB and EmbA using ClustalW (Figure 2) shows 30\% sequence identity; two segments of this comparison are shown previously indicating in bold letters the residues interacting with ethambutol in Figure 1. If they have this sequence identity, and the structure of proteins is more conserved than sequences, the structure of this segment in all Emb should be very similar and points to the fact that ethambutol possibly binds to the same site in EmbB and EmbA. The amino acid residues that in our model interact with Ethambutol, 740, 1052, and 1055, are conserved in EmbC, EmbA, and EmbB; nevertheless residue 1051, an aspartic acid in EmbC, hydrogen bonded to Ethambutol, is not conserved in EmbA (D1051G) or in EmbB (D1051R). The electrostatic and van der Waals energy contribution of this residue should change the binding energy between Ethambutol and the C-terminal domain in the other members of this family of enzymes. This position in the binding site deserves attention for improvement and design of modified compounds effective against resistant strains.

\section{Conclusions}

Our study provides a structural hypothesis for the binding modes of ethambutol to the C-terminal domain of the arabinosyltransferase of Mycobacterium tuberculosis, EmbC. The analysis of these binding models suggest that amino acid residues ASH1051, ASN740, ASP1052, and ARG1055 play a key role in the binding of the ethambutol to C-terminal domain EmbC. Due to the conservation of the amino acid residues in the binding site, it is possible that Ethambutol binds to the same site in EmbA and EmbB.

\section{Acknowledgments}

This work has been partially supported by CIMAV, SC, and Consejo Nacional de Ciencia y Tecnología (CONACYT, Mexico). D. G. Mitnik is a researcher of CONACYT and CIMAV. This work was also partially supported by operating Grant no. 01/2011 from the Universidad Católica de la Santísima Concepcion (Chile). This work has been partially supported by Fondecyt 1130707.

\section{References}

[1] R. Goude, A. G. Amin, D. Chatterjee, and T. Parish, "The arabinosyltransferase EmbC is inhibited by ethambutol in Mycobacterium tuberculosis," Antimicrobial Agents and Chemotherapy, vol. 53, no. 10, pp. 4138-4146, 2009.

[2] A. B. Bloch, I. M. Onorato, and K. G. Castro, "Multipleantibiotic-resistant bacteria," The New England Journal of Medicine, vol. 331, no. 10, pp. 678-679, 1994.

[3] D. B. Kitchen, H. Decornez, J. R. Furr, and J. Bajorath, "Docking and scoring in virtual screening for drug discovery: methods and applications," Nature Reviews, vol. 3, no. 11, pp. 935-949, 2004.

[4] GLIDE, Maestro, version 9.1, Schrodinger, New York, NY, USA, 2010.

[5] R. A. Friesner, J. L. Banks, R. B. Murphy et al., "Glide: a new approach for rapid, accurate docking and scoring. 1. Method and assessment of docking accuracy," Journal of Medicinal Chemistry, vol. 47, no. 7, pp. 1739-1749, 2004.

[6] T. A. Halgren, R. B. Murphy, R. A. Friesner et al., "Glide: a new approach for rapid, accurate docking and scoring. 2. Enrichment factors in database screening," Journal of Medicinal Chemistry, vol. 47, no. 7, pp. 1750-1759, 2004.

[7] Prime Version 3.1, Schrödinger, New York, NY, USA, 2012. 
[8] J. A. Luke, S. L. Georgina, G. Hemza et al., "The C-terminal domain of the arabinosyltransferase Mycobacterium tuberculosis EmbC is a lectin-like carbohydrate binding module," PLoS Pathogens, vol. 7, no. 2, Article ID e1001299, 2011. 

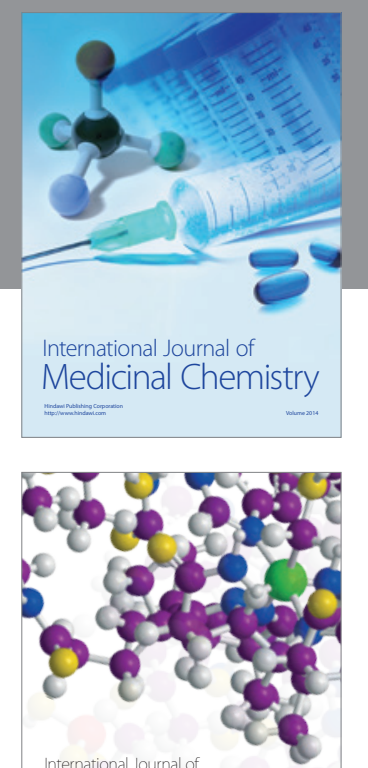

\section{Carbohydrate} Chemistry

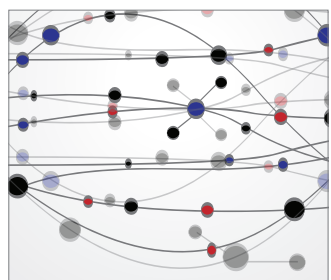

The Scientific World Journal
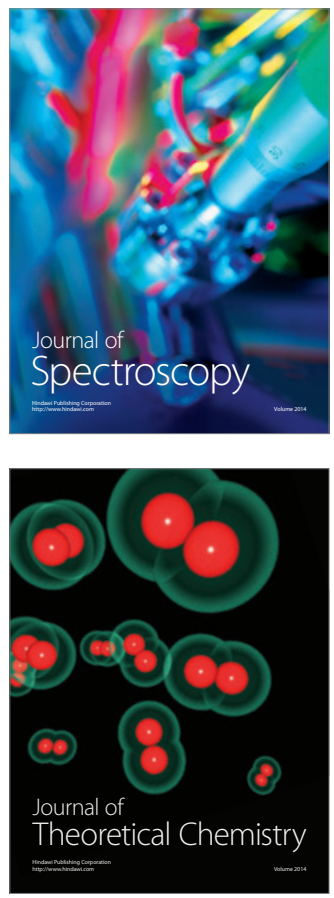
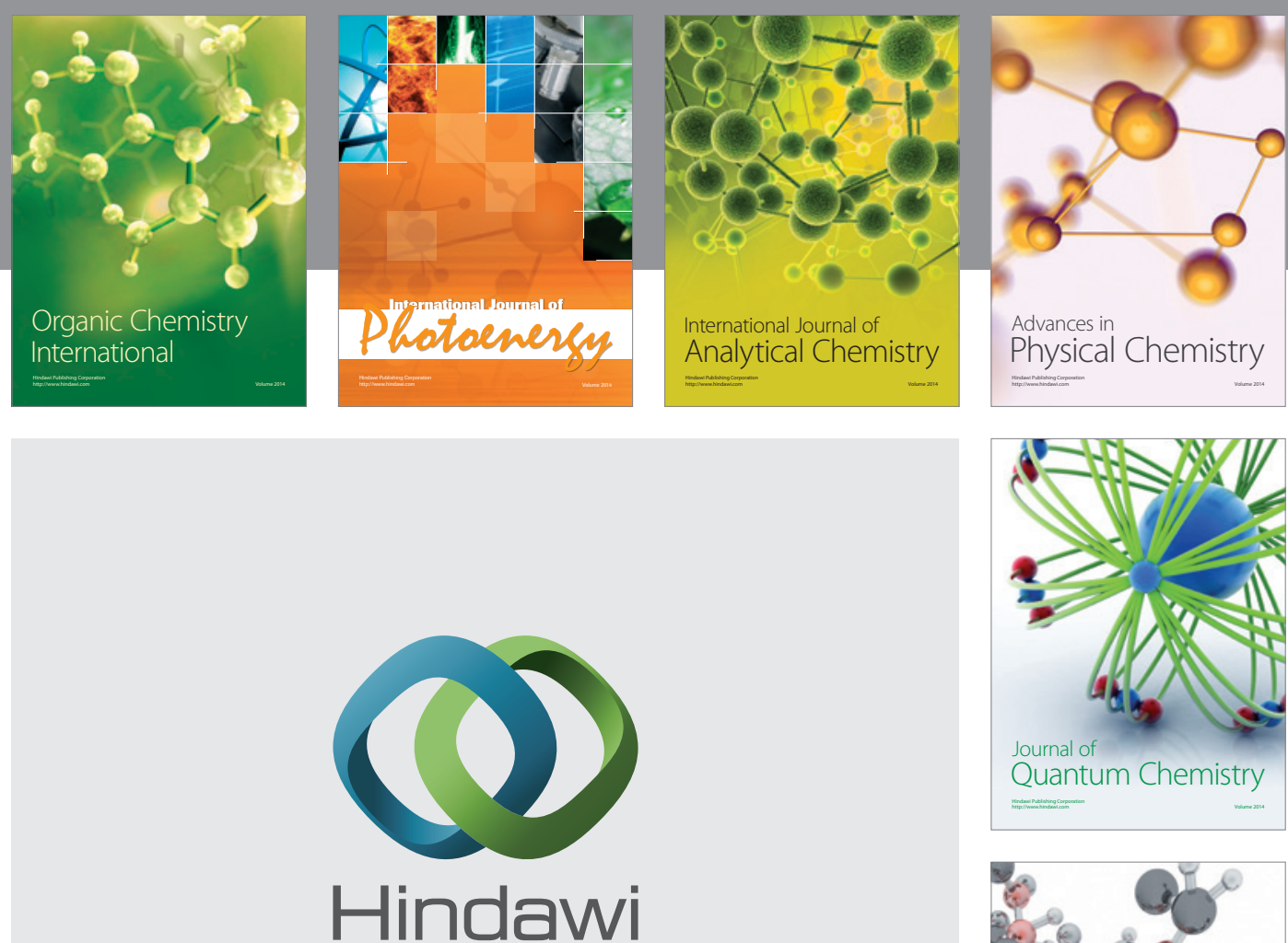

Submit your manuscripts at

http://www.hindawi.com

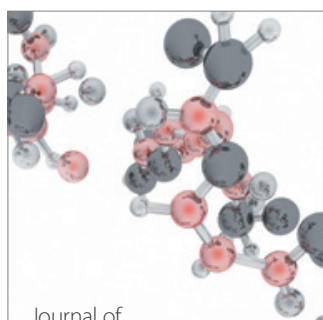

Analytical Methods

in Chemistry

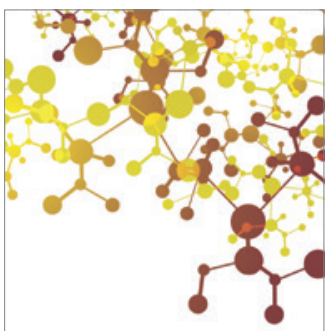

Journal of

Applied Chemistry

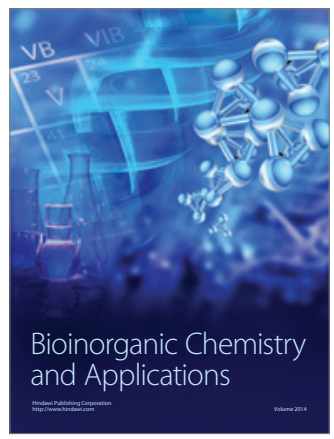

Inorganic Chemistry
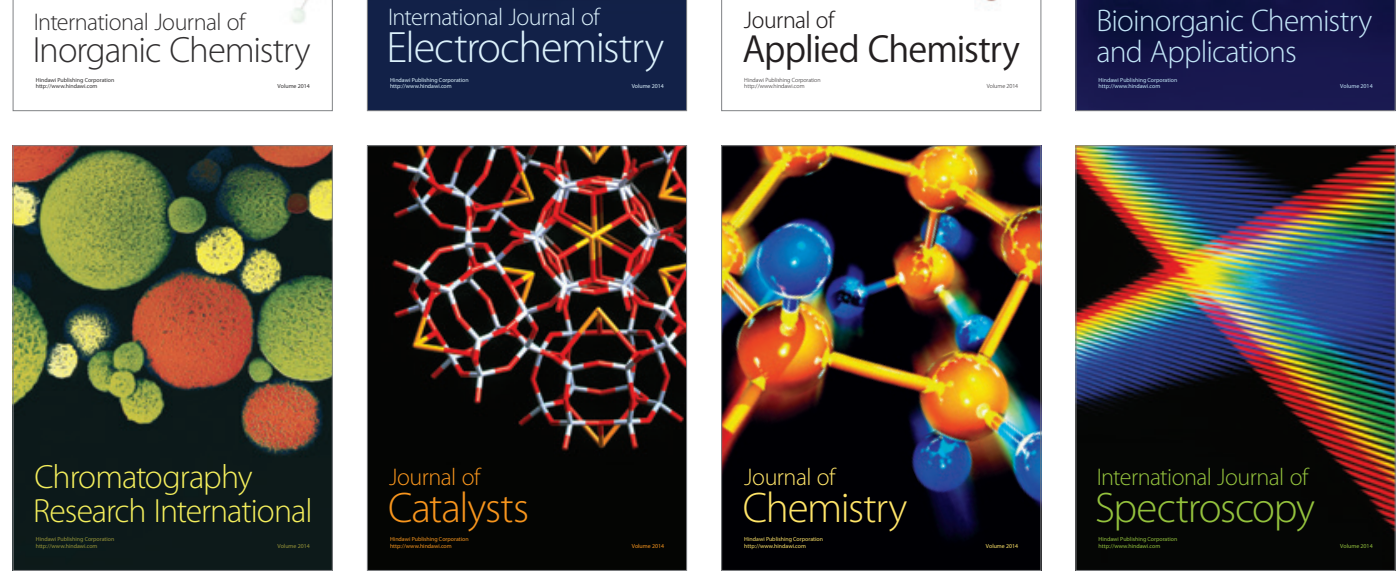